\title{
Protein Kinases and Phosphatases of the Plastid and Their Potential Role in Starch Metabolism
}

\author{
Chris White-Gloria', Jayde J. Johnson' ${ }^{1}$, Kayla Marritt' ${ }^{1}$, Amr Kataya ${ }^{1,2}$, Ahmad Vahab' \\ and Greg B. Moorhead ${ }^{1 *}$
}

${ }^{1}$ Department of Biological Sciences, University of Calgary, Calgary, AB, Canada, ${ }^{2}$ Department of Chemistry and Biosciences, University of Stavanger, Stavanger, Norway

Phospho-proteomic studies have confirmed that phosphorylation is a common mechanism to regulate protein function in the chloroplast, including the enzymes of starch metabolism. In addition to the photosynthetic machinery protein kinases (STN7 and STN8) and their cognate protein phosphatases PPH1 (TAP38) and PBCP, multiple other protein kinases and phosphatases have now been localized to the chloroplast. Here, we build a framework for understanding protein kinases and phosphatases, their regulation, and potential roles in starch metabolism. We also catalog mapped phosphorylation sites on proteins of chloroplast starch metabolism to illustrate the

OPEN ACCESS

Edited by:

Michael James Emes,

University of Guelph, Canada

Reviewed by:

Alan M. Myers,

lowa State University, United States

Jean-David Rochaix,

Université de Genève, Switzerland

*Correspondence:

Greg B. Moorhead moorhead@ucalgary.ca

Specialty section:

This article was submitted to

Plant Metabolism

and Chemodiversity,

a section of the journal

Frontiers in Plant Science

Received: 20 March 2018

Accepted: 25 June 2018

Published: 17 July 2018

Citation:

White-Gloria C, Johnson JJ, Marritt K,

Kataya A, Vahab A and Moorhead GB

(2018) Protein Kinases

and Phosphatases of the Plastid

and Their Potential Role in Starch

Metabolism. Front. Plant Sci. 9:1032.

doi: 10.3389/fpls.2018.01032 potential and mostly unknown roles of protein phosphorylation in the regulation of starch biology.

Keywords: chloroplast, protein phosphatase, casein kinase 2, TAP38, PP2C, SLP1, STN7, STN8

\section{INTRODUCTION}

Mass spectrometry, and particularly quantitative mass spectrometry, has further established the prevalence of protein covalent modifications as a mechanism to control protein function (Baginsky and Gruissem, 2009; Grabsztunowicz et al., 2017; Hartl et al., 2017). Although ubiquitination and acetylation are emerging as common modifications, phosphorylation by protein kinases is still recognized as the most common protein covalent modification and is found universally across the domains of life (Adam and Hunter, 2017; Hartl et al., 2017). Quantitative mass spectrometry has made a conservative estimate that $75 \%$ of all human proteins are regulated by protein phosphorylation (Baginsky and Gruissem, 2009; Sharma et al., 2014). Protein kinases are one of the largest super-families in all Eukaryotes and in conjunction with phospho-proteomic studies from a variety of Eukaryotes (including plants) it is thought that protein phosphorylation is likely as common in other Eukaryotes as it is in humans. Currently there are $\sim 1050$ and $\sim 150$ protein kinases and phosphatases annotated, respectively, in the Arabidopsis thaliana genome (Uhrig et al., 2013b).

Although up to 9 amino acids can be modified by phosphorylation, the majority of protein phosphorylation occurs on serine, threonine, and tyrosine and this is true for plants as well as other Eukaryotes (van Wijk et al., 2014; Adam and Hunter, 2017). Notably, several Eukaryotic histidine kinases and phosphatases have recently been discovered (Fuhs and Hunter, 2017). Although phosphorylation of plant proteins on tyrosine is now widely accepted, there is some debate as to whether chloroplast proteins are tyrosine phosphorylated. A recent re-examination of mass spectrometry data could not conclude phosphorylation on tyrosine for plastid proteins (Lu et al., 2015b; Baginsky, 2016). Until stronger evidence is brought forward, 
we will work with the assumption that there is no protein tyrosine phosphorylation in chloroplasts (Lu et al., 2015b; Baginsky, 2016). In addition to protein phosphorylation, the reversible formation of disulphide bonds (i.e., redox regulation) on plastid proteins is well documented (Lehtimaki et al., 2015; Grabsztunowicz et al., 2017). Recent work has also confirmed widespread protein acetylation (Hartl et al., 2017), and instances of protein methylation, glycosylation, nitration and nitrosylation, sumoylation, and glutathionylation in chloroplasts (Grabstunowicz et al., 2017).

It is generally accepted that the 'players' or proteins of starch synthesis and degradation have been elucidated, yet our understanding of pathway regulation is far from complete (Kotting et al., 2010; Pfister and Zeeman, 2016). A full understanding of regulation of pathway enzymes (and other proteins) will undoubtedly involve allosteric effectors (metabolites) and covalent modifications. We will use this review to highlight the abundance of protein phosphorylation of starch metabolic enzymes and the potential machinery involved in these modifications. Readers should be constantly aware that regulation by protein phosphorylation will not operate in isolation and will ultimately need to be considered in relation to other covalent modifications.

\section{PROTEIN PHOSPHORYLATION IN THE CHLOROPLAST}

Given the abundance of protein phosphorylation in prokaryotes it is no surprise that an organelle derived from prokaryotes (plastids) has many events controlled by this process. Consistent with this are the growing proteomic datasets that demonstrate widespread protein phosphorylation in the chloroplast. Bennett (1977) demonstrated for the first time, protein phosphorylation within the chloroplast and identified 26 and $9 \mathrm{kDa}$ thylakoid membrane proteins as phosphoproteins. Soon after this, a light and redox sensitive protein kinase was found to be responsible for this event and subsequently identified in Chlamydomonas reinhardtii as serine/threonine-protein kinase 7 (Stt7) with its ortholog in Arabidopsis being STN7 (Depege et al., 2003; Bellafiore et al., 2005). Thylakoid bound STN7 and the chloroplast and sequence related protein kinase STN8, each have unique, and some overlapping substrates (Schonberg et al., 2017), with STN7 required for light harvesting complex II (LHCII) phosphorylation and state transitions and STN8 for photosystem II (PSII) core protein phosphorylation (Bonardi et al., 2005; Vainonen et al., 2005). We refer the reader to numerous other reviews on chloroplast protein phosphorylation (Baginsky and Gruissem, 2009; Schonberg and Baginsky, 2012; Baginsky, 2016; Grieco et al., 2016; Grabsztunowicz et al., 2017).

We will refer to data assembled in the PhosPhAt database ${ }^{1}$ but remind readers to be cautious about information collated from multiples studies. Many of these mass spectrometry studies do not report false discovery rates (FDRs) for peptide

${ }^{1}$ phosphat.uni-hohenheim.de/ identification, there can be wrongly assigned phosphorylation sites, and most of these studies are not quantitative, and thus no information of phosphorylation stoichiometry is known. Due to the sensitivity of mass spectrometry, many of these sites will have very low stoichiometry, but this may also reflect the dynamic nature of covalent modifications and a vast array of conditions that experiments were performed under. We refer readers to excellent discussions of this data in ( $\mathrm{Lu}$ et al., 2015b; Baginsky, 2016). Using the entire PhosPhAt 4.0 dataset, as many as 800 chloroplast phospho-proteins are reported for Arabidopsis thaliana (Baginsky, 2016). Using only three studies with reported FDRs less than 1\%, reduces this number to 427 chloroplast phospho-proteins, and this includes many starch metabolic enzymes. Our analysis here of the starch metabolic machinery utilized the entire PhosPhAt 4.0 dataset with all tyrosine phosphorylation sites removed (for reasons stated above). This information (Supplementary Table 1) should be regarded as a start point for a study and all sites should ultimately be confirmed by additional research. Instances of specific sites being identified in multiple studies increases support for that being a correctly identified site. As PhosPhAt is the most comprehensive plant phosphoproteomic database and is primarily derived from photosynthetic tissue, we will build our discussion around chloroplast starch metabolism (transient starch). We will also discuss several works on amyloplast protein phosphorylation and refer to smaller phospho-proteomic datasets for maize and rice amyloplasts which are linked to individual published articles (Nakagami et al., 2010; Facette et al., 2013; Hou et al., 2015; Lu et al., 2015a).

\section{THE PLAYERS: PLASTID PROTEIN PHOSPHATASES, KINASES AND THE STARCH ENZYMATIC MACHINERY}

It has been commented that the discovery of new chloroplast protein kinases has stagnated recently and this likely indicates that the catalog is nearing completion (Richter et al., 2016). A similar comment can reasonably be applied to the chloroplast protein phosphatases (Uhrig and Moorhead, 2011). With the inventory being near completion we can now start to utilize phospho-proteomic data and mapped sites on starch metabolic enzymes to tease out potential protein kinase/phosphatase substrates using genetics and biochemistry utilizing this list of players.

\section{Starch Enzymatic Machinery}

We have utilized the PhosPhAt 4.0 database to explore phosphorylation of the starch enzymatic machinery (Kotting et al., 2010; Pfister and Zeeman, 2016) and present this information in Supplementary Table 1 . We have only included sites mapped by mass spectrometry (not predicted sites) and acknowledge that all studies have some degree of FDR and possibly incorrectly assigned phospho-amino acids. All data (Supplementary Table 1) are with respect to Arabidopsis thaliana, come from a variety of tissues (mostly rosettes) and metabolic 
conditions. The original publications leading to the PhosPhAT dataset are found on the website. Although glucan waterdikinase 2 or GWD2 (cytosolic), $\beta$-amylase 5 (cytosolic), $\beta$-amylase 7 (nuclear) and $\beta$-amylase 8 (nuclear) are phosphoproteins, they are excluded from this table because they are not plastid localized (Pfister and Zeeman, 2016) and thus do not play a direct role in starch synthesis and degradation in this organelle. Several enzymes are marked 'none' to indicate they have yet to be shown to be phospho-proteins, but this may only reflect the depth of the studies, tissue used, metabolic conditions, or workflow (for instance, granule bound enzymes could be lost in a first step of a phospho-proteomic workflow).

\section{A Compilation of Phosphorylation Sites}

The first notable feature of this table is that most starch machinery proteins are phospho-proteins, and most are phosphorylated at multiple sites, including chloroplast transit peptides (cTP), with phosphorylation predominantly on serine and threonine residues [we have removed several potential phospho-tyrosines based on Lu et al. (2015b) and Baginsky (2016)]. Protein kinases phosphorylate specific amino acids based on distinct motifs around the phospho-site, making analysis of compiled phosphorylation sites potentially revealing. A sequence logo (Figure 1) of the sites from Supplementary Table 1 show that phosphorylation of the starch metabolic machinery is likely not carried out by only one or two protein kinases, but it suggests that many protein kinases, likely controlled (activated/ inactivated) by differing metabolic or environmental conditions, impinge on these enzymes. What does emerge from the data are motifs indicating a proline directed kinase (note SP or SerPro sites), a casein kinase [prevalence of acidic residues around the phosphosite (Schonberg et al., 2014; Lu et al., 2015a)] and likely one or both of STN7 and STN8 [G or Gly before the phosphosite (Schonberg et al., 2017)] phosphorylate the starch metabolic machinery. Although 4 casein kinase 2 catalytic subunits exist in Arabidopsis, only CK2 $\alpha 4$ is plastid localized (Salinas et al., 2006).

\section{Plastid Protein Kinases}

In addition to the well-characterized thylakoid bound protein kinases STN7 and 8, other chloroplast protein kinases have been identified and include casein kinase $2 \alpha 4$ (CK2 $\alpha 4$ ), three thylakoid-associated kinases (TAKs), chloroplast sensor kinase (CSK), a family of atypical protein kinases (Activity of $\mathrm{BC} 1$ Complex Kinase- or $\mathrm{ABC} 1 \mathrm{~K}$ ), and most recently Plastid Protein Kinase With Unknown Function [PKU1, PKU2, PKU3, PKU5, and PKU12 (also ABC1K9)] were described (Snyders and Kohorn, 1999; Schliebner et al., 2008; Baginsky and Gruissem, 2009; Baginsky, 2016; Richter et al., 2016). Evidence exists for $\mathrm{Ca}^{2+}$-dependent chloroplast protein phosphorylation; however, identification of $\mathrm{Ca}^{2+}$-dependent protein kinases has eluded researchers to date (Makhmoudova et al., 2014; Baginsky, 2016), as does a proline directed protein kinase as suggested by Figure 1, Supplementary Table 1, and (Baginsky, 2016). As phosphorylation consensus motifs are lacking for several of these

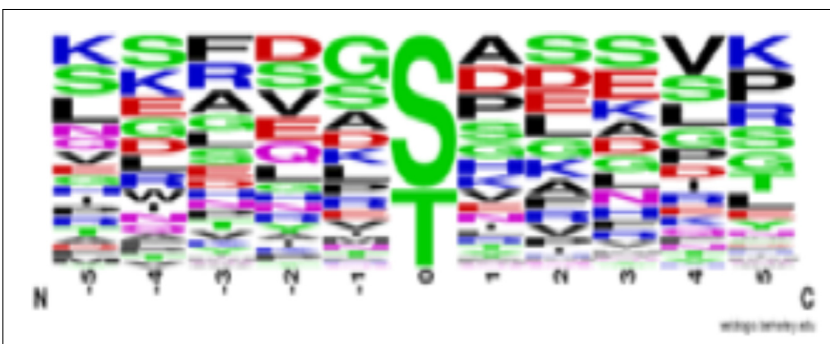

FIGURE 1 | Sequence logo of phosphorylation sites identified in the starch metabolic machinery of Arabidopsis thaliana (see Supplementary Table 1). Sequences are centered on protein phosphorylation sites (position 0) and were gathered from PhosPhAt database 4.0 (http://phosphat.uni-hohenheim. de/phosphat.html). Phosphorylation sites located in chloroplast transit peptides (cTP) were excluded in the figure. Letter size indicates prevalence of amino acid at a given position. Sequence alignment was created using WebLogo (https://weblogo.berkeley.edu/logo.cgi).

protein kinases we cannot infer if any of these kinases potentially phosphorylate the sites in Supplementary Table 1 (Figure 1).

The Eukaryotic protein kinases all likely evolved from a single ancestral gene (Manning et al., 2002a,b; Moorhead et al., 2007, 2009) and contain the domains defined in Manning et al. (2002b). Typically, protein kinases are activated by phosphorylation in their activation-, or T-loop sequence, and inactivated by dephosphorylation of the same site, allowing for the turning on and off of substrate phosphorylation. Phosphorylation brings about a series of conformational changes in the active site that typically activate the enzyme 50 - to 100 -fold. Of the plastid protein kinases mentioned above, only the TAK1-3 enzymes appear to have classic activation loops and appear to be phosphorylated at the appropriate loop site to potentially activate the enzyme (Figure 2). CSK and PKUs are of prokaryotic origin and based on sequence do not have activation loops (CSK) or clearly defined activation loops (PKUs). The PKUs appear to have four of the twelve conserved eukaryotic protein kinase domains, including a 'DFG' motif (see Richter et al., 2016). CSK is a histidine kinase and although it binds ATP with high affinity, it may not phosphorylate target proteins (Ibrahim et al., 2016). Variations in putative activation loops and protein kinase domains warrants biochemical studies to confirm if these enzymes display true protein kinase activity or not. Interestingly, CK2 $\alpha 4$, STN7, and STN8 all have a phosphomimetic glutamate (E) at the appropriate or equivalent position in their activation loops and likely exist in a constitutively active form (Figure 2; Lolli et al., 2017). Included in this alignment of activation loops is human CK2 $\alpha$ and phosphorylase kinase $\gamma(\mathrm{PhK} \gamma$, the catalytic subunit) which have the equivalent $\mathrm{E}$ in their activation loops. $\mathrm{PhK} \gamma$ is considered constitutively active and is regulated by additional subunits and $\mathrm{CK} 2 \alpha$ is considered non-conventional and always in the active conformation (Lolli et al., 2017). CK2 enzymes also have additional regulatory $\beta$ subunits, but no plant $\beta$ subunit appears to reside in the plastid (Salinas et al., 2006). Having three of the major chloroplast protein kinases 'always active' has implications for regulation of phosphorylation events. Notably, data does exist indicating that chloroplast CK2 $\alpha 4$ and 
STN7 activity is redox regulated (Schonberg and Baginsky, 2012; Baginsky, 2016; Shapiguzov et al., 2016).

STN 7 and 8 were initially characterized to phosphorylate thylakoid associated proteins. Intuitively, it is hard to rationalize phosphorylation of 'soluble' stromal enzymes by thylakoid bound protein kinases, yet a recent phosphoproteomic study identified several non-thylakoid proteins as substrates of these thylakoid bound enzymes (Schonberg et al., 2017). That study confirmed the preference for glycine $(G)$ at -1 , as is seen in several of the starch machinery protein phospho-sites. In addition, preferences at $-1,+1,+2$, and +3 are also in several of the potential targets listed in Supplementary Table 1 building that case that STN7 and STN8 target several starch players (Schonberg et al., 2017). Multiple sites in several Supplementary Table 1 proteins also fit the CK2 consensus motif (Schonberg et al., 2014). The phosphoproteomic datasets complied in PhosPhAt support the idea that the major stromal (soluble) protein kinase of chloroplasts is CK2 $\alpha 4$ and corroborate other studies implicating CK2 $\alpha 4$ as a regulator of plastid gene expression, RNA stability, fatty acid biosynthesis, the Calvin cycle, and energy metabolism, in addition to starch metabolism (Baginsky and Gruissem, 2009; Reiland et al., 2009). The large number of SP sites found in Supplementary Table 1 strongly supports the role of a prolinedirected kinase as a regulator of the starch machinery, but to date, no plastid kinase has been characterized that has a phosphorylation consensus that fits this motif description. Interestingly, Makhmoudova et al. (2014) have biochemically identified and partially purified two $\mathrm{Ca}^{2+}$-dependent protein kinases from maize amyloplast extracts that phosphorylate starch branching enzyme IIb (SBEIIb).

\section{Plastid Protein Phosphatases}

Unlike protein kinases, it is thought that Eukaryotic protein phosphatases evolved independently four times being reflected by the four sequence unique families known as the phosphoprotein phosphatases (PPP), the $\mathrm{Mg}^{2+} / \mathrm{Mn}^{2+}$-dependent enzymes (PP2C/PPM), the protein tyrosine phosphatases (PTP) and the aspartate-based enzymes (Uhrig et al., 2013b). The major serine/threonine phosphatases are the PPP 1-7 that collectively are responsible for $\sim 80-90 \%$ of all serine/threonine protein dephosphorylation in Eukaryotic cells (Moorhead et al., 2007; Heroes et al., 2013). With the exception of PP2B (PP3), all other PPP members are conserved in plants (Kerk et al., 2008; Meekins et al., 2015), yet none have been plastid localized based on early work utilizing biochemical assays and more recently informatics (MacKintosh et al., 1991; Uhrig et al., 2013b).

The PPP protein phosphatase catalytic subunit can be regarded as a catalytic engine, although specificity for serine/threonine versus tyrosine exists within the "catalytic machine." Free PPP catalytic subunits display promiscuous activity in vitro and achieve substrate specificity and regulation by association with additional proteins or regulatory subunits (Moorhead et al., 2009; Templeton et al., 2011; Uhrig et al., 2013b; Labandera et al., 2015). Genomics has identified several new members for the PPP-family (BSU1, SLP1, SLP2, and RLPH2), all of which are present in plants (Uhrig et al., 2013a,b), but not all Eukaryotes. All PPP members were considered

hPKA
hPKc
hCDK2
hCaMKI
hMMPKa
hMAPKK (MEK)
hPhRy
hCK2a
CK2a4
sTN7
STN8
TAK1
TAK2
TAK3

FIGURE 2 | Alignment of the activation or T-loops of multiple human and Arabidopsis thaliana chloroplast protein kinases. Well characterized human (h) protein kinases were selected and activation loops defined by the motifs DFG and APE (red) are shown. For the human protein kinases (h), the serine or threonine shown in bold and underlined is the known (phospho)-amino acid that is phosphorylated or dephosphorylated to activate and inactivate the kinase, respectively. The bold and underlined threonines (TT) of TAK1-3 have been shown to be phospho-residues by mass spectrometry analysis, suggesting they are activated in the same fashion. Human phosphorylase kinase $(\mathrm{PhK} \gamma)$ is not phosphorylated in its T-loop, but like CK2 $\alpha$ is in a constitutively active conformation and has a negatively charged $\mathrm{E}$ in its T-loop that fulfills the role of a phospho-amino acid. An equivalent $E$ is found in plant CK2 (CK2 $\alpha 4)$ and the thylakoid protein kinases STN7 and 8 (bold, underlined, and green).

serine/threonine specific until the recent biochemical analysis of SLP1, SLP2, and RLPH2, which display (at least some) activity against phospho-tyrosine (Uhrig and Moorhead, 2011; Uhrig et al., 2016, 2017; Labandera et al., 2018). Important for this discussion, SLP1 is chloroplast localized. Like other PPP enzymes, it is expected that SLP1 will have additional binding partners to control its activity, but to date, none have been identified. Biochemical analysis of SLP1 revealed it is found in no other location except chloroplasts, is expressed in both light and dark, is insensitive to the classic PPP family inhibitors microcystin and okadaic acid and is particularly sensitive to inhibition by free phosphate. Sensitivity to phosphate inhibition is within chloroplast phosphate concentrations and may link SLP1 activity to changing free phosphate concentrations in light/dark transitions (Uhrig and Moorhead, 2011).

The PP2C enzymes (also called PPM) are serine/threonine specific and have proliferated in plants with 80 annotated in Arabidopsis thaliana (compared to 20 in humans) (Shi, 2009; Fuchs et al., 2013; Chen et al., 2017). PPH1 and PBCP were identified as key protein phosphatases that control the phospho-status of LHCII proteins and PSII and are thought to counter kinases STN7 and STN8, respectively (Pribil et al., 2010; Shapiguzov et al., 2010; Samol et al., 2012). PPH1 is also known as TAP38, and along with PBCP belongs to the PP2C (PPM) family of phosphatases (Pribil et al., 2010; Kerk et al., 2015). Other PP2C enzymes (Table 1) have been localized to chloroplasts. Schliebner et al. (2008) used bioinformatics to predict cTPs in Arabidopsis protein kinases and phosphatases. Using this information, they formally demonstrated 6 different PP2C cTPs could localize red 
TABLE 1 | Chloroplastic protein phosphatases of Arabidopsis thaliana.

\begin{tabular}{|c|c|c|c|c|}
\hline Name & AGI code (or GenBank) & Type & Localization & Reference \\
\hline PR1 & AT4G21210 & Phosphotransferase & Chloroplast & Chastain et al., 2008 \\
\hline SLP1 & AT1G07010 & PPP & Chloroplast & Uhrig and Moorhead, 2011 \\
\hline PP2C & AT1G07160 & PP2C & Chloroplast & Schliebner et al., 2008 \\
\hline AP2C1 & AT2G30020 & PP2C & Chloroplast & Schliebner et al., 2008 \\
\hline PP2C62 & AT4G33500 & PP2C & Chloroplast & Schliebner et al., 2008 \\
\hline PP2C14 & AT1G67820 & PP2C & Chloroplast & Schliebner et al., 2008 \\
\hline PBCP & AT2G30170 & PP2C & Chloroplast & Puthiyaveetil et al., 2014 \\
\hline TAP38/PPH1 & AT4G27800 & PP2C & Chloroplast & Pribil et al., 2010; Shapiguzov et al., 2010 \\
\hline PP2C52 & AT4G03415 & PP2C & Chloroplast & Schliebner et al., 2008 \\
\hline
\end{tabular}

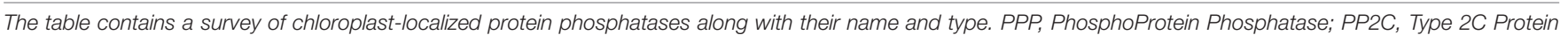
Phosphatase. PR1 is a phosphotranferase and technically not a phosphatase.

fluorescent protein (RFP) to the chloroplast, strongly suggesting the endogenous enzymes reside there. To date, no biological functions for these $6 \mathrm{PP} 2 \mathrm{Cs}$ are known. We refer readers to Box 1 of Uhrig et al. (2013b) for a more detailed history and naming of the serine/threonine protein phosphatases. To date, no specific protein phosphatase has been linked to the starch machinery of plastids.

Protein tyrosine phosphatase family biochemistry has determined that many proteins placed in the PTP group based on sequence are in fact not protein phosphatases, but dephosphorylate other molecules (glycogen, starch, mRNA, and phosphoinositides) (Moorhead et al., 2007; Tonks, 2013; Silver et al., 2014). The best characterized examples of this in plants being the starch phosphatases SEX4, Like-SEX4-1 (LSF1), and 2 (LSF2) (Silver et al., 2014; Gentry et al., 2016). Few phospho-tyrosine specific phosphatases have been identified in plants (Uhrig et al., 2016), and none have been plastid localized. We refer readers to Box 1 of Silver et al. (2014) for details on PTP family nomenclature, regulation, and substrate specificity.

\section{PROTEIN PHOSPHORYLATION AND STARCH METABOLISM}

\section{Phosphorylation of Starch Metabolic Enzymes-Maize Endosperm as a Model}

Although we have focused on the starch machinery of Arabidopsis photosynthetic tissue, several key works on the phosphorylation of these proteins comes from the endosperm (storage starch) of maize. Multiple phospho-proteomic and focused studies have demonstrated phosphorylation of many starch synthesis and degradative enzymes underscoring the key role of this protein covalent modification in starch biology (Tetlow et al., 2004; Crofts et al., 2017). It is unclear in most cases which protein kinase or phosphatase controls these events and importantly what the consequence of protein phosphorylation is on the biological activity of individual enzymes. Seminal work by Tetlow et al. (2004) first showed protein phosphorylation played a vital role in the formation of a starch-synthesizing protein complex composed of SBEIIb and SBEI as complex formation was phosphorylation dependent. Furthermore, Tetlow et al. (2004) showed that phosphorylation contributes to the regulation of SBEII isoform catalytic activity in both chloroplasts and amyloplasts.

Starch metabolic enzymes can be soluble in the stroma or bound to the starch granule- either surface associated, or within the granule. Grimaud et al. (2008) performed a (phospho)proteomic study of enzymes bound within the granule. Using a phospho-binding dye (Pro-Diamond Q) they demonstrated that granule bound starch synthase (GBSS), SBEIIb and starch phosphorylase [PHS1 (Pho1 in other species)] were phosphoproteins. The role of phosphorylation was not explored, nor was proteomics used to identify lower abundance proteins in the granule. Clearly, it would be interesting to see if protein kinases or phosphatases reside within, or on the surface of the granule to perform their job, or if proteins get phosphorylated in the stroma for recruitment and/or altering of activity. More recently, Makhmoudova et al. (2014) explored the phosphorylation of SBEIIb, one of the highly phosphorylated proteins detected in Grimaud et al. (2008). This study was important for several reasons. First, an amyloplast stromal fraction, in the presence of $\gamma$ - ${ }^{32} \mathrm{P}$-ATP, could readily label multiple proteins in the extract, consistent with phospho-proteomic studies discussed above. Second, they identified 3 phosphorylation sites on SBEIIb and perhaps most importantly, they uncovered two peaks of $\mathrm{Ca}^{2+}$ dependent protein kinase activity. The identify of these protein kinases and the function of SBEIIb phosphorylation has yet to be resolved.

\section{CONCLUSION AND FUTURE DIRECTIONS}

\section{What Do All of These Phosphorylation Sites Mean?}

Protein phosphorylation is prevalent in the plastid and clearly the starch machinery is controlled in this fashion-typically at multiple sites and with multiple protein kinases suggesting multiple factors/conditions feed into regulating these enzymes. How does phosphorylation affect these proteins? It could alter enzyme activity, control protein-protein interactions, localization in the cell, protein turnover, or even association with starch. All of these 
questions coupled with the observed degree of phosphorylation of the starch machinery really tell us we are still just at the tip of the iceberg in terms of understanding the role of protein kinases and phosphatases in starch synthesis and degradation.

\section{Where Now?}

Protein kinases have been more studied than protein phosphatases for multiple factors, with one key reason being that in vitro, protein kinases display substrate specificity based on short sequence motifs, and phosphatases, in general, do not. This meant discovering protein phosphatase substrates using a biochemical approach has been hampered and results from this approach often confusing (Cohen, 1994). The recent advent of quantitative phospho-proteomics has opened a new chapter in substrate discovery for protein phosphatases and protein kinases (Bian et al., 2013; Wang et al., 2013; Rusin et al., 2015, 2017). In principle, quantitative mass spectrometry will allow a direct comparison of wild-type plants (tissues or cells) to a knock out line in, for instance, a specific protein phosphatase, and uncover phosphorylated substrates that accumulate in the absence of the phosphatase under some condition or stress. Similarly, loss of a protein kinase should tease out specific phosphorylation sites on a substrate (i.e., a quantitative loss). This was proven effective to identify putative substrates of a human PP4 complex in the DNA damage response (Lee et al., 2012) and PP6 in mitosis (Rusin et al., 2015) and for multiple protein kinases (Bian et al., 2013; Wang et al., 2013; Hou et al., 2015; Rusin et al., 2017). However, readers should keep in mind potential pitfalls of this approach. For instance, one protein kinase may normally phosphorylate and activate another protein kinase. Loss of the upstream protein kinase would then result in loss of substrate phosphorylation for the downstream kinase (which is now not activated) and it would appear that these are substrates of the upstream kinase. That said, quantitative phospho-proteomics likely represents the new focal point of plant protein kinase and phosphatase

\section{REFERENCES}

Adam, K., and Hunter, T. (2017). Histidine kinases, and the missing phosphoproteome from prokaryotes to eukaryotes. Lab. Invest. 98, 233-247. doi: 10.1038/labinvest.2017.118

Baginsky, S. (2016). Protein phosphorylation in chloroplasts - a survey of phosphorylation targets. J. Exp. Bot. 67, 3873-3882. doi: 10.1093/jxb/erw098

Baginsky, S., and Gruissem, W. (2009). The chloroplast kinase network: new insights from large-scale phosphoproteome profiling. Mol. Plant 2, 1141-1153. doi: $10.1093 / \mathrm{mp} / \mathrm{ssp} 058$

Bellafiore, S., Barneche, F., Peltier, G., and Rochaix, J. D. (2005). Rochaix, State transitions and light adaptation require chloroplast thylakoid protein kinase STN7. Nature 433, 892-895. doi: 10.1038/nature03286

Bennett, J. (1977). Phosphorylation of chloroplast membrane polypeptides. Nature 269, 344-346. doi: 10.1038/269344a0

Bian, Y., Ye, M., Wang, C., Cheng, K., Song, C., Dong, M., et al. (2013). Global screening of CK2 kinase substrates by an integrated phosphoproteomics workflow. Sci. Rep. 3:3460. doi: 10.1038/srep03460

Bonardi, V., Pesaresi, P., Becker, T., Schleiff, E., Wagner, R., Pfannschmidt, T., et al. (2005). Photosystem II core phosphorylation and photosynthetic acclimation require two different protein kinases. Nature 437, 1179-1182. doi: 10.1038/ nature 04016

Chastain, C. J., Xu, W., Parsley, K., Sarath, G., Hibberd, J. M., and Chollet, R. (2008). The pyruvate, orthophosphate dikinase regulatory proteins substrate identification. Coupled with biochemistry and genetics, this should usher in a new era in protein phosphorylation research, including uncovering roles in plant starch synthesis and degradation. As stated before, we must always remember that protein phosphorylation does not operate in isolation in the cell and is coordinated with (potentially) multiple other covalent modifications.

\section{AUTHOR CONTRIBUTIONS}

CW-G, JJ, KM, AK, AV, and GM all participated in the writing of this review.

\section{FUNDING}

GM was supported by the Natural Sciences and Engineering Research Council of Canada and AK was funded by the Research Council of Norway grant number 251310/F20.

\section{ACKNOWLEDGMENTS}

The authors would like to thank other past and present lab members that have contributed our understanding of chloroplast protein phosphatases. They also thank Shane Emmanuelle for discussions on SnRKs and starch metabolism.

\section{SUPPLEMENTARY MATERIAL}

The Supplementary Material for this article can be found online at: https://www.frontiersin.org/articles/10.3389/fpls.2018.01032/ full\#supplementary-material

of Arabidopsis possess a novel, unprecedented Ser/Thr protein kinase primary structure. Plant J. 53, 854-863. doi: 10.1111/j.1365-313X.2007.03 366.x

Chen, M. J., Dixon, J. E., and Manning, G. (2017). Genomics and evolution of protein phosphatases. Sci. Signal. 10:eaag1796. doi: 10.1126/scisignal.aag1796

Cohen, P. (1994). The discovery of protein phosphatases: from chaos and confusion to an understanding of their role in cell regulation and human disease. Bioessays 16, 583-588. doi: 10.1002/bies.950160812

Crofts, N., Nakamura, Y., and Fujita, N. (2017). Critical and speculative review of the roles of multi-protein complexes in starch biosynthesis in cereals. Plant Sci. 262, 1-8. doi: 10.1016/j.plantsci.2017.05.007

Depege, N., Bellafiore, S., and Rochaix, J. D. (2003). Role of chloroplast protein kinase Stt7 in LHCII phosphorylation and state transition in Chlamydomonas. Science 299, 1572-1575. doi: 10.1126/science.1081397

Facette, M. R., Shen, Z., Bjornsdottir, F. R., Briggs, S. P., and Smith, L. G. (2013). Parallel proteomic and phosphoproteomic analyses of successive stages of maize leaf development. Plant Cell 25, 2798-2812. doi: 10.1105/tpc.113.11 2227

Fuchs, S., Grill, E., Meskiene, I., and Schweighofer, A. (2013). Type 2C protein phosphatases in plants. FEBS J. 280, 681-693. doi: 10.1111/j.1742-4658.2012. 08670.x

Fuhs, S. R., and Hunter, T. (2017). pHisphorylation: the emergence of histidine phosphorylation as a reversible regulatory modification. Curr. Opin. Cell Biol. 45, 8-16. doi: 10.1016/j.ceb.2016.12.010 
Gentry, M. S., Brewer, M. K., and Vander Kooi, C. W. (2016). Structural biology of glucan phosphatases from humans to plants. Curr. Opin. Struct. Biol. 40, 62-69. doi: 10.1016/j.sbi.2016.07.015

Grabsztunowicz, M., Koskela, M. M., and Mulo, P. (2017). Post-translational modifications in regulation of chloroplast function: recent advances. Front. Plant Sci. 8:240. doi: 10.3389/fpls.2017.00240

Grieco, M., Jain, A., Ebersberger, I., and Teige, M. (2016). An evolutionary view on thylakoid protein phosphorylation uncovers novel phosphorylation hotspots with potential functional implications. J. Exp. Bot. 67, 3883-3896. doi: 10.1093/ jxb/erw164

Grimaud, F., Rogniaux, H., James, M. G., Myers, A. M., and Planchot, V. (2008). Proteome and phosphoproteome analysis of starch granule-associated proteins from normal maize and mutants affected in starch biosynthesis. J. Exp. Bot. 59, 3395-3406. doi: 10.1093/jxb/ern198

Hartl, M., Fussl, M., Boersema, P. J., Jost, J. O., Kramer, K., Bakirbas, A., et al. (2017). Lysine acetylome profiling uncovers novel histone deacetylase substrate proteins in Arabidopsis. Mol. Syst. Biol. 13:949. doi: 10.15252/msb.20177819

Heroes, E., Lesage, B., Gornemann, J., Beullens, M., Van Meervelt, L., and Bollen, M. (2013). The PP1 binding code: a molecular-lego strategy that governs specificity. FEBS J. 280, 584-595. doi: 10.1111/j.1742-4658.2012.08547.x

Hou, Y., Qiu, J., Tong, X., Wei, X., Nallamilli, B. R., Wu, W., et al. (2015). A comprehensive quantitative phosphoproteome analysis of rice in response to bacterial blight. BMC Plant Biol. 15:163. doi: 10.1186/s12870-015-0541-2

Ibrahim, I. M., Puthiyaveetil, S., Khan, C., and Allen, J. F. (2016). Probing the nucleotide-binding activity of a redox sensor: two-component regulatory control in chloroplasts. Photosynth. Res. 130, 93-101. doi: 10.1007/s11120-0160229-y

Kerk, D., Silver, D., Uhrig, R. G., and Moorhead, G. B. (2015). PP2C7s", genes most highly elaborated in photosynthetic organisms, reveal the bacterial origin and stepwise evolution of PPM/PP2C protein phosphatases. PLoS One 10:e132863. doi: 10.1371 /journal.pone.0132863

Kerk, D., Templeton, G., and Moorhead, G. B. (2008). Evolutionary radiation pattern of novel protein phosphatases revealed by analysis of protein data from the completely sequenced genomes of humans, green algae, and higher plants. Plant Physiol. 146, 351-367. doi: 10.1104/pp.107.111393

Kotting, O., Kossmann, J., Zeeman, S. C., and Lloyd, J. R. (2010). Regulation of starch metabolism: the age of enlightenment? Curr. Opin. Plant Biol. 13, 321-329. doi: 10.1016/j.pbi.2010.01.003

Labandera, A. M., Uhrig, R. G., Colville, K., Moorhead, G. B., and Ng, K. K. S. (2018). Structural basis for the preference of the Arabidopsis thaliana phosphatase RLPH2 for tyrosine-phosphorylated substrates. Sci. Signal. 11:eaan8804. doi: 10.1126/scisignal.aan8804

Labandera, A. M., Vahab, A. R., Chaudhuri, S., Kerk, D., and Moorhead, G. B. (2015). The mitotic PP2A regulator ENSA/ARPP-19 is remarkably conserved across plants and most eukaryotes. Biochem. Biophys. Res. Commun. 458, 739-744. doi: 10.1016/j.bbrc.2015.01.123

Lee, D. H., Goodarzi, A. A., Adelmant, G. O., Pan, Y., Jeggo, P. A., Marto, J. A., et al. (2012). Phosphoproteomic analysis reveals that PP4 dephosphorylates KAP-1 impacting the DNA damage response. EMBO J. 31, 2403-2415. doi: $10.1038 /$ emboj.2012.86

Lehtimaki, N., Koskela, M. M., and Mulo, P. (2015). Posttranslational modifications of chloroplast proteins: an emerging field. Plant Physiol. 168, 768-775. doi: 10.1104/pp.15.00117

Lolli, G., Naressi, D., Sarno, S., and Battistutta, R. (2017). Characterization of the oligomeric states of the CK2 alpha2beta2 holoenzyme in solution. Biochem. J. 474, 2405-2416. doi: 10.1042/BCJ20170189

Lu, Q., Ding, S., Reiland, S., Rodiger, A., Roschitzki, B., Xue, P., et al. (2015a). Identification and characterization of chloroplast casein kinase II from Oryza sativa (rice). J. Exp. Bot. 66, 175-187. doi: 10.1093/jxb/eru405

Lu, Q., Helm, S., Rodiger, A., and Baginsky, S. (2015b). On the extent of tyrosine phosphorylation in chloroplasts. Plant Physiol. 169, 996-1000. doi: 10.1104/pp. 15.00921

MacKintosh, C., Coggins, J., and Cohen, P. (1991). Plant protein phosphatases. Subcellular distribution, detection of protein phosphatase $2 \mathrm{C}$ and identification of protein phosphatase $2 \mathrm{~A}$ as the major quinate dehydrogenase phosphatase. Biochem. J. 273(Pt 3), 733-738.

Makhmoudova, A., Williams, D., Brewer, D., Massey, S., Patterson, J., Silva, A., et al. (2014). Identification of multiple phosphorylation sites on maize endosperm starch branching enzyme IIb, a key enzyme in amylopectin biosynthesis. J. Biol. Chem. 289, 9233-9246. doi: 10.1074/jbc.M114.551093

Manning, G., Plowman, G. D., Hunter, T., and Sudarsanam, S. (2002a). Evolution of protein kinase signaling from yeast to man. Trends Biochem. Sci. 27, 514-520.

Manning, G., Whyte, D. B., Martinez, R., Hunter, T., and Sudarsanam, S. (2002b). The protein kinase complement of the human genome. Science 298, 1912-1934. doi: $10.1126 /$ science. 1075762

Meekins, D. A., Raththagala, M., Auger, K. D., Turner, B. D., Santelia, D., Kotting, O., et al. (2015). Mechanistic insights into glucan phosphatase activity against polyglucan substrates. J. Biol. Chem. 290, 23361-23370. doi: 10.1074/jbc. M115.658203

Moorhead, G. B., De Wever, V., Templeton, G., and Kerk, D. (2009). Evolution of protein phosphatases in plants and animals. Biochem. J. 417, 401-409. doi: 10.1042/BJ20081986

Moorhead, G. B., Trinkle-Mulcahy, L., and Ulke-Lemee, A. (2007). Emerging roles of nuclear protein phosphatases. Nat. Rev. Mol. Cell Biol. 8, 234-244. doi: $10.1038 / \mathrm{nrm} 2126$

Nakagami, H., Sugiyama, N., Mochida, K., Daudi, A., Yoshida, Y., Toyoda, T., et al. (2010). Large-scale comparative phosphoproteomics identifies conserved phosphorylation sites in plants. Plant Physiol. 153, 1161-1174. doi: 10.1104/pp. 110.157347

Pfister, B., and Zeeman, S. C. (2016). Formation of starch in plant cells. Cell. Mol. Life Sci. 73, 2781-2807. doi: 10.1007/s00018-016-2250-x

Pribil, M., Pesaresi, P., Hertle, A., Barbato, R., and Leister, D. (2010). Role of plastid protein phosphatase TAP38 in LHCII dephosphorylation and thylakoid electron flow. PLoS Biol. 8:e1000288. doi: 10.1371/journal.pbio.1000288

Puthiyaveetil, S., Woodiwiss, T., Knoerdel, R., Zia, A., Wood, M., Hoehner, R., et al. (2014). Significance of the photosystem II core phosphatase PBCP for plant viability and protein repair in thylakoid membranes. Plant Cell Physiol. 55, 1245-1254. doi: 10.1093/pcp/pcu062

Reiland, S., Messerli, G., Baerenfaller, K., Gerrits, B., Endler, A., Grossmann, J., et al. (2009). Large-scale Arabidopsis phosphoproteome profiling reveals novel chloroplast kinase substrates and phosphorylation networks. Plant Physiol. 150, 889-903. doi: 10.1104/pp.109.138677

Richter, A. S., Gartmann, H., Fechler, M., Rodiger, A., Baginsky, S., and Grimm, B. (2016). Identification of four plastid-localized protein kinases. FEBS Lett. 590, 1749-1756. doi: 10.1002/1873-3468.12223

Rusin, S. F., Adamo, M. E., and Kettenbach, A. N. (2017). Identification of candidate casein kinase 2 substrates in mitosis by quantitative phosphoproteomics. Front. Cell Dev. Biol. 5:97. doi: 10.3389/fcell.2017.00097

Rusin, S. F., Schlosser, K. A., Adamo, M. E., and Kettenbach, A. N. (2015). Quantitative phosphoproteomics reveals new roles for the protein phosphatase PP6 in mitotic cells. Sci. Signal. 8:rs12. doi: 10.1126/scisignal.aab3138

Salinas, P., Fuentes, D., Vidal, E., Jordana, X., Echeverria, M., and Holuigue, L. (2006). An extensive survey of CK2 alpha and beta subunits in Arabidopsis: multiple isoforms exhibit differential subcellular localization. Plant Cell Physiol. 47, 1295-1308. doi: 10.1093/pcp/pcj100

Samol, I., Shapiguzov, A., Ingelsson, B., Fucile, G., Crevecoeur, M., Vener, A. V., et al. (2012). Identification of a photosystem II phosphatase involved in light acclimation in Arabidopsis. Plant Cell 24, 2596-2609. doi: 10.1105/tpc.112. 095703

Schliebner, I., Pribil, M., Zuhlke, J., Dietzmann, A., and Leister, D. (2008). A survey of chloroplast protein kinases and phosphatases in Arabidopsis thaliana. Curr. Genomics 9, 184-190. doi: 10.2174/138920208784340740

Schonberg, A., and Baginsky, S. (2012). Signal integration by chloroplast phosphorylation networks: an update. Front. Plant Sci. 3:256. doi: 10.3389/fpls. 2012.00256

Schonberg, A., Bergner, E., Helm, S., Agne, B., Dunschede, B., Schunemann, D., et al. (2014). The peptide microarray "ChloroPhos1.0" identifies new phosphorylation targets of plastid casein kinase II (pCKII) in Arabidopsis thaliana. PLoS One 9:e108344. doi: 10.1371/journal.pone.0108344

Schonberg, A., Rodiger, A., Mehwald, W., Galonska, J., Christ, G., Helm, S., et al. (2017). Identification of STN7/STN8 kinase targets reveals connections between electron transport, metabolism and gene expression. Plant J. 90, 1176-1186. doi: 10.1111/tpj.13536

Shapiguzov, A., Chai, X., Fucile, G., Longoni, P., Zhang, L., and Rochaix, J. D. (2016). Activation of the Stt7/STN7 kinase through dynamic interactions with 
the cytochrome b6f complex. Plant Physiol. 171, 82-92. doi: 10.1104/pp.15. 01893

Shapiguzov, A., Ingelsson, B., Samol, I., Andres, C., Kessler, F., Rochaix, J. D., et al. (2010). The PPH1 phosphatase is specifically involved in LHCII dephosphorylation and state transitions in Arabidopsis. Proc. Natl. Acad. Sci. U.S.A. 107, 4782-4787. doi: 10.1073/pnas.0913810107

Sharma, K., D’Souza, R. C., Tyanova, S., Schaab, C., Wisniewski, J. R., Cox, J., et al. (2014). Ultradeep human phosphoproteome reveals a distinct regulatory nature of Tyr and Ser/Thr-based signaling. Cell Rep. 8, 1583-1594. doi: 10.1016/ j.celrep.2014.07.036

Shi, Y. (2009). Serine/threonine phosphatases: mechanism through structure. Cell 139, 468-484. doi: 10.1016/j.cell.2009.10.006

Silver, D. M., Kotting, O., and Moorhead, G. B. (2014). Phosphoglucan phosphatase function sheds light on starch degradation. Trends Plant Sci. 19, 471-478. doi: 10.1016/j.tplants.2014.01.008

Snyders, S., and Kohorn, B. D. (1999). TAKs, thylakoid membrane protein kinases associated with energy transduction. J. Biol. Chem. 274, 9137-9140. doi: $10.1074 /$ jbc.274.14.9137

Templeton, G. W., Nimick, M., Morrice, N., Campbell, D., Goudreault, M., Gingras, A. C., et al. (2011). Identification and characterization of AtI-2, an Arabidopsis homologue of an ancient protein phosphatase 1 (PP1) regulatory subunit. Biochem. J. 435, 73-83. doi: 10.1042/BJ20101035

Tetlow, I. J., Wait, R., Lu, Z., Akkasaeng, R., Bowsher, C. G., Esposito, S., et al. (2004). Protein phosphorylation in amyloplasts regulates starch branching enzyme activity and protein-protein interactions. Plant Cell 16, 694-708. doi: $10.1105 /$ tpc. 017400

Tonks, N. K. (2013). Protein tyrosine phosphatases - from housekeeping enzymes to master regulators of signal transduction. FEBS J. 280, 346-378. doi: 10.1111/ febs. 12077

Uhrig, R. G., Kerk, D., and Moorhead, G. B. (2013a). Evolution of bacteria-like phosphoprotein phosphatases in photosynthetic eukaryotes features ancestral mitochondrial or archaeal origin and possible lateral gene transfer. Plant Physiol. 163, 1829-1843. doi: 10.1104/pp.113.224378

Uhrig, R. G., Labandera, A. M., and Moorhead, G. B. (2013b). Arabidopsis PPP family of serine/threonine protein phosphatases: many targets but few engines. Trends Plant Sci. 18, 505-513. doi: 10.1016/j.tplants.2013.05.004
Uhrig, R. G., Labandera, A. M., Muhammad, J., Samuel, M., and Moorhead, G. B. (2016). Rhizobiales-like phosphatase 2 from Arabidopsis thaliana is a novel phospho-tyrosine-specific phospho-protein phosphatase (PPP) family protein phosphatase. J. Biol. Chem. 291, 5926-5934. doi: 10.1074/jbc.M115.68 3656

Uhrig, R. G., Labandera, A. M., Tang, L. Y., Sieben, N. A., Goudreault, M., Yeung, E., et al. (2017). Activation of mitochondrial protein phosphatase SLP2 by MIA40 regulates seed germination. Plant Physiol. 173, 956-969. doi: 10.1104/ pp.16.01641

Uhrig, R. G., and Moorhead, G. B. (2011). Two ancient bacterial-like PPP family phosphatases from Arabidopsis are highly conserved plant proteins that possess unique properties. Plant Physiol. 157, 1778-1792. doi: 10.1104/pp.111.18 2493

Vainonen, J. P., Hansson, M., and Vener, A. V. (2005). STN8 protein kinase in Arabidopsis thaliana is specific in phosphorylation of photosystem II core proteins. J. Biol. Chem. 280, 33679-33686. doi: 10.1074/jbc.M505729200

van Wijk, K. J., Friso, G., Walther, D., and Schulze, W. X. (2014). Meta-analysis of Arabidopsis thaliana phospho-proteomics data reveals compartmentalization of phosphorylation motifs. Plant Cell 26, 2367-2389. doi: 10.1105/tpc.114.12 5815

Wang, P., Xue, L., Batelli, G., Lee, S., Hou, Y. J., Van Oosten, M. J., et al. (2013). Quantitative phosphoproteomics identifies SnRK2 protein kinase substrates and reveals the effectors of abscisic acid action. Proc. Natl. Acad. Sci. U.S.A. 110, 11205-11210. doi: 10.1073/pnas.1308974110

Conflict of Interest Statement: The authors declare that the research was conducted in the absence of any commercial or financial relationships that could be construed as a potential conflict of interest.

Copyright (c) 2018 White-Gloria, Johnson, Marritt, Kataya, Vahab and Moorhead. This is an open-access article distributed under the terms of the Creative Commons Attribution License (CC BY). The use, distribution or reproduction in other forums is permitted, provided the original author(s) and the copyright owner(s) are credited and that the original publication in this journal is cited, in accordance with accepted academic practice. No use, distribution or reproduction is permitted which does not comply with these terms. 